\title{
Estimativa da pegada de carbono no setor de soja no município de Paragominas, Pará
}

A pegada de carbono surgiu com o intuito de administrar as emissões de gases de efeito estufa e é uma das várias formas de se medir seus impactos, obtendo grande visibilidade na comunidade acadêmica após o vislumbre de seus resultados. Objetiva-se, portanto, utilizar indicador de pegada de carbono para estimar as emissões de GEE geradas na produção de soja no município de Paragominas-PA, no período de 1997 a 2013, e com isso avaliar a contribuição dessa cultura agrícola para o balanço de emissões e as mudanças climáticas regionais. Realizou-se o cálculo da pegada de carbono com dados de volume de produção e área plantada de soja no município de Paragominas e após isso utilizou-se a metodologia de Cheng et al. (2011) para aferir seu valor. Tal metodologia realiza o cálculo da Pegada de Carbono total a partir da somatória das pegadas de carbono individuais por uso de fertilizantes, da eletricidade usada na irrigação, do uso de agrotóxicos e das emissões de N2O. Analisando os resultados referentes à Pegada de Carbono anual, percebe-se que ela vem obtendo valores mais significativos com o passar do tempo em virtude principalmente do aumento da área plantada de soja. Infere-se ainda que o valor de PC está mais ligado à esta área plantada do que propriamente à produção de soja, isto porque é durante a plantação que são empregados os fertilizantes e agrotóxicos - refletidos nos valores de PCF e PCA - e a área total de produção influencia diretamente os valores de PCl e indiretamente as outras Pegadas individuais.

Palavras-chave: Indicador Ambiental; Sustentabilidade; Agricultura.

\section{Estimate of carbon footprint of soy sector in the city Paragominas, Para}

\begin{abstract}
The carbon footprint came up with the intention of managing emissions of greenhouse gases and is one of several ways to measure their impact, achieving high visibility in the academic community after the glimmer of its results. The objective of this paper, therefore, is to use carbon footprint indicator to estimate the GHG emissions generated in soybean production in the city of Paragominas-PA in the period 19972013, and thus evaluate the contribution of this crop for the balance of emissions and regional climate change. We carried out the calculation of the carbon footprint with production volume data and soy planted area in the city of Paragominas and thereafter used the method of Cheng et al. (2011) to assess its value. This methodology calculates the total Carbon Footprint from the sum of individual carbon footprints by using fertilizers, electricity used for irrigation, use of pesticides and N2O emissions. Analyzing the results of the annual carbon footprint, it is clear that it has achieved the most significant values over time due primarily to increased planted area of soybeans. It is inferred even if the PC value is more connected to this acreage than to the production of soybeans, this is because fertilizers and pesticides are used during planting reflected in the PCF and PCA values - and the total area production directly influences the PCl values and indirectly other individual footprints.
\end{abstract}

Keywords: Environmental Indicator; Sustainability; Agriculture.

Topic: Indicadores de Sustentabilidade

Reviewed anonymously in the process of blind peer.

Renan Coelho de Vasconcellos

Universidade do Estado do Pará

http://lattes.cnpq.br/5840345105754478

renanrcv@gmail.com

Norma Ely Santos Beltrão

Justus-Liebig Universität Giessen, JLU, Alemanha

http://lattes.cnpq.br/9434131723316393

normaelybeltrao@gmail.com

Altem Nascimento Pontes

Universidade Estadual de Campinas

http://lattes.cnpq.br/5993352890364998

altempontes@hotmail.com

DOI: 10.6008/SPC2179-6858.2016.001.0012
Received: $\mathbf{3 0 / 1 0 / 2 0 1 5}$

Approved: 30/11/2015
Referencing this:

VASCONCELLOS, R. C.; BELTRÃO, N. E. S.; PONTES, A. N.. Estimativa da pegada de carbono no setor de soja no município de Paragominas, Pará. Revista Ibero-Americana de Ciências Ambientais, v.7, n.1, p.142-149, 2016. DOI: http://doi.org/10.6008/SPC2179$\underline{6858.2016 .001 .0012}$ 


\section{INTRODUÇÃO}

As mudanças climáticas ocasionadas pela emissão antrópica de gases de efeito estufa (GEE), vêm nos últimos tempos ganhando espaço em debates envolvendo cientistas e tomadores de decisão, a exemplo do Painel Intergovernamental de Mudanças Climáticas (IPCC) (PANDEY et al., 2011). Devido à sensibilidade apresentada por atividades relacionadas à agricultura, o aumento de temperatura global é recebido com preocupação por empreendimentos desta área, em virtude de poder ocasionar estresse hídrico e incidência de pragas e doenças (GROSSI et al., 2010).

A pegada de carbono (PC) surgiu com o intuito de administrar as emissões de GEE e é uma das várias formas de se medir seus impactos, obtendo grande visibilidade na comunidade acadêmica após o vislumbre de seus resultados (CARBON TRUST, 2012; PANDEY et al., 2011). Hertwich e Peters (2009) apontam a importância desta forma de estimativa para diminuir os níveis de poluição e servir de indicador ambiental em diversas atividades, haja vista que quantifica o impacto negativo da emissão de carbono.

O valor da pegada de carbono pode ser entendido como uma Análise do Ciclo de Vida das emissões de carbono e de outros gases de efeito estufa que ocorrem ao longo de uma determinada cadeia produtiva e são utilizadas como forma de avaliação de sustentabilidade (CUCEK et al., 2012). Cabe ressaltar que existem diversos tipos de "pegadas", contudo a pegada de carbono é a mais importante nos últimos anos como forma de indicador de conservação ambiental (GALLI et al., 2012). Conforme explanado por Montibeller et al. (2012), o uso de instrumentos econômicos - tal como a pegada de carbono - auxiliam aos tomadores de decisão a adquirirem um ponto de vista mais socioambiental, contrapondo-se a visão usual de "crescimento em desfavor do meio ambiente".

Atualmente, a soja ganha grande relevância no setor agronegócio do Brasil, e grande parte disso deve-se às exportações deste produto, a sua elevada produtividade, aos baixos custos de produção e a expansão desenfreada da sua "fronteira" na Amazônia (FAGUNDES et al., 2013). No Pará, a soja se desenvolve ao se aproveitar de vantagens comparativas como a facilidade de escoamento da produção, incentivos fiscais do Governo do Estado, infraestrutura de estradas e energia elétrica (LEÃO \& SOUZA, 2012).

A demanda internacional se faz presente principalmente para alimentação animal, alimentos e outros usos, tais como biodiesel. Graças ao elevado índice de exportação nacional, o Brasil se tornou o segundo maior produtor de soja mundial em 2010 e 2011, com cerca de 73,8 milhões de toneladas. Cabe ressaltar que um dos motivos desta expressiva produtividade foi a expansão das áreas de cultivo no período entre 1995 a 2011 (CASTANHEIRA \& FREIRE, 2013).

Tendo em vista não somente a expansão da soja no Estado do Pará, mas os potenciais impactos locais, regionais e globais, dada a associação desta expansão com a mudanças de uso da terra na Amazônia, propõe-se a utilização do indicador de pegada de carbono para estimar as emissões de GEE geradas na produção de soja no município de Paragominas-PA, no período de 1997 a 2013, e com isso avaliar a contribuição dessa cultura agrícola para o balanço de emissões e as mudanças climáticas regionais. 


\section{METODOLOGIA}

\section{Etapas da pesquisa}

A escolha da área de estudo ocorreu a partir da constatação de que o município de Paragominas é o que apresenta maior produtividade de soja no Estado do Pará, segundo IBGE (2013). A primeira etapa da pesquisa se deu a partir de levantamento bibliográfico. Sua importância foi de se conhecer a temática a ser estudada, avaliando outros trabalhos que tratam sobre Pegada de Carbono e procurando um modelo de cálculo que pudesse ser utilizado para atingir os objetivos propostos.

Como etapa posterior, realizou-se a garimpagem de dados em IBGE (2013) para delimitação do volume de produção e área plantada de soja no município de Paragominas dentre o período de 1997 a 2013. Devido à ausência de números referentes a fertilizantes e agrotóxicos específicos para a soja no município, optou-se por consultar os valores médios de utilização destes produtos em kg/ha no Estado do Pará, a partir de IBGE (2012). Não foram coletados dados mais recentes por ainda não estarem presentes na base de informações do IBGE.

\section{Análise dos dados}

Diversas metodologias de cálculo de pegada de carbono no setor agrícola podem ser encontradas na literatura científica, mas de modo geral se baseiam em três escopos: i) emissões de carbono ocasionadas pela mudança do uso do solo (DON et al., 2011; GUO \& GIFFORD, 2002; HOUGHTON et al., 2012; POST \& KWON, 2000); ii) emissões diretas por fontes de queima de combustíveis fósseis e insumos agrícolas (CHENG et al., 2011; KAHRL et al., 2010; WEST \& MARLAND, 2003); e iii) emissões provenientes do carbono estocado no solo e tipo de manejo (BAYER et al. 2004; CONCEIÇÃO et al., 2013; VEZZANI \& MIELNICZUK, 2011; WEST \& MARLAND, 2003)

Conforme destacado por Castanheira e Freire (2013), o cálculo de pegada de carbono aplicado ao setor da soja ainda apresenta alguns fatores que podem gerar imprecisões no seu resultado. Tais fatores são: i) dificuldade de exatidão no cálculo de emissões pelo solo; e ii) tipo de manejo do solo em questão.

Contudo, uma estimativa com foco no auxílio à tomada de decisão em áreas consideravelmente grandes pode se tornar inviável caso necessite destes dados primários, haja vista que encarece o cálculo na medida que precisa de coleta de amostras e análises laboratoriais para alcançar o resultado mais preciso. Tal visão corrobora com um dos diversos preceitos a respeito da construção e utilização de indicadores, o de que eles devem ser de fácil mensuração (COSTA, 2013).

Analisando-se, ainda, estudos a respeito de emissões de carbono por uso de insumos agrícolas e queima de combustíveis fósseis, tais como Cheng et al. (2011), Kahrl et al. (2010) e West \& Marland (2003), percebe-se que apresentam estimativas satisfatórias e que podem auxiliar o processo de governança no tocante às mudanças climáticas. Em se tratando do cálculo de emissões pela parcela de carbono no solo, percebe-se pelos trabalhos de Bayer et al. (2004), Conceição et al. (2013); Vezzani \& Mielniczuk (2011) e 
West \& Marland (2003) que o foco maior é dado à estocagem de carbono no solo pelos determinados tipos de cultura e não às suas emissões subsuperficiais.

Tendo em vista tais considerações, adotou-se o posicionamento neste trabalho de se utilizar o escopo de análise de emissões oriundas dos insumos de produção agrícola. O modelo de cálculo para a análise de dados foi baseado no utilizado por Cheng et al. (2011) e se dá a partir da utilização da seguinte fórmula:

$$
P C=P C F+P C I+P C N+P C A .
$$

Aqui, $P C$ é a pegada de carbono total; $P C F$ é a pegada de carbono oriunda do uso de fertilizantes; $P C I$ é a pegada de carbono da eletricidade utilizada para irrigação; $P C N$ é a pegada de carbono das emissões de $\mathrm{N}_{2} \mathrm{O}$; e $P C A$ é a pegada de carbono advinda do uso de agrotóxicos. Todos os valores individuais nada mais são do que a multiplicação dos dados de entrada pelos fatores. Esses fatores também são os utilizados por Cheng et al. (2011) e são listados na Tabela 1.

Tabela 1: Fatores de Emissão por tipo de Pegada individual.

\begin{tabular}{cc}
\hline Pegada individual & Fator de emissão \\
\hline PCF & 1,74 ton de $\mathrm{C} /$ ton de fertilizante $\mathrm{N}$ \\
& 0,2 ton de $\mathrm{C} /$ ton de fertilizante $\mathrm{P}$ \\
PCN & 0,15 ton de $\mathrm{C} /$ ton de fertilizante $\mathrm{K}$ \\
PCA & 0,01 ton de $\mathrm{N}_{2} \mathrm{O} /$ ton de fertilizante $\mathrm{N}$ \\
PCl & 4,93 ton de $\mathrm{C} /$ ton de total de agrotóxicos \\
\hline
\end{tabular}

Fonte: Cheng et al. (2011).

Cabe ressaltar que, apesar da elevada taxa de precipitação no município de Paragominas, a qual pode tornar desnecessário o uso de irrigação pelo fato dela ser maior que a quantidade necessária suprir a deficiência hídrica no cultivo de soja (BISPO, 2007), ainda sim há uma redução de tal taxa em alguns períodos do ano (PACHECO et al., 2004; SANTOS, 2008), os quais eventualmente podem necessitar de irrigação para manter a produção.

Um segundo indicador foi elaborado para que se possa ter uma melhor visualização da pegada de carbono. Tal indicador é a intensidade de carbono, consistindo na divisão do valor de $P C$ pela quantidade total de soja produzida no ano analisado.

\section{RESULTADOS E DISCUSSÕES}

O cálculo de Pegada de Carbono e intensidade de carbono por quantidade produzida de soja para o município de Paragominas, no período de 1997 a 2013, foram feitos do modo exposto na metodologia e são apresentados na Tabela 2.

Analisando os resultados referentes à Pegada de Carbono anual, percebe-se que ela vem obtendo valores mais significativos com o passar do tempo em virtude principalmente do aumento da área plantada de soja. Infere-se ainda que o valor de $P C$ está mais ligado à esta área plantada do que propriamente à produção de soja, isto porque é durante a plantação que são empregados os fertilizantes e agrotóxicos refletidos nos valores de $P C F$ e $P C A$ - e a área total de produção influencia diretamente os valores de $P C l$ e indiretamente as outras Pegadas individuais. 
Tal fato é evidenciado ao verificar os valores de área plantada e produção total de soja nos anos de 1998 e 1999. Ambos os anos apresentaram produção em torno de 840 ton, contudo o primeiro teve uma área plantada e valor de $P C$ maiores que o ano seguinte. Esta análise é facilitada ao conferir a Figura 1, a qual demonstra as variações de $P C$ em relação à área plantada.

Tabela 2: Estimativa de Pegada de Carbono em Paragominas.

\begin{tabular}{ccccc}
\hline Ano & Área (ha) & $\begin{array}{c}\text { Quantidade produzida } \\
\text { (ton) }\end{array}$ & $\begin{array}{c}\text { Pegada de carbono } \\
\text { (ton) }\end{array}$ & $\begin{array}{c}\text { Intensidade de carbono por quantidade } \\
\text { produzida (tonC/ton) }\end{array}$ \\
\hline 1997 & 325 & 877 & 11.44975 & 0.013056 \\
1998 & 920 & 846 & 32.4116 & 0.038312 \\
1999 & 500 & 840 & 17.615 & 0.02097 \\
2000 & 1540 & 1155 & 54.2542 & 0.046973 \\
2001 & 280 & 754 & 9.8644 & 0.013083 \\
2002 & 1120 & 3326 & 39.4576 & 0.011863 \\
2003 & 3000 & 8640 & 105.69 & 0.012233 \\
2004 & 3259 & 9777 & 114.8146 & 0.011743 \\
2005 & 6990 & 20970 & 246.2577 & 0.011743 \\
2006 & 10000 & 30000 & 352.3 & 0.011743 \\
2007 & 6000 & 21000 & 211.38 & 0.010066 \\
2008 & 11720 & 35160 & 412.8956 & 0.011743 \\
2009 & 14200 & 42600 & 500.266 & 0.011743 \\
2010 & 26600 & 71820 & 937.118 & 0.013048 \\
2011 & 37348 & 119514 & 1315.77 & 0.011009 \\
2012 & 35354 & 123740 & 1245.521 & 0.010066 \\
2013 & 42000 & 121800 & 1479.66 & 0.012148 \\
\hline
\end{tabular}

Fonte: Autores (2015), IBGE (2013).

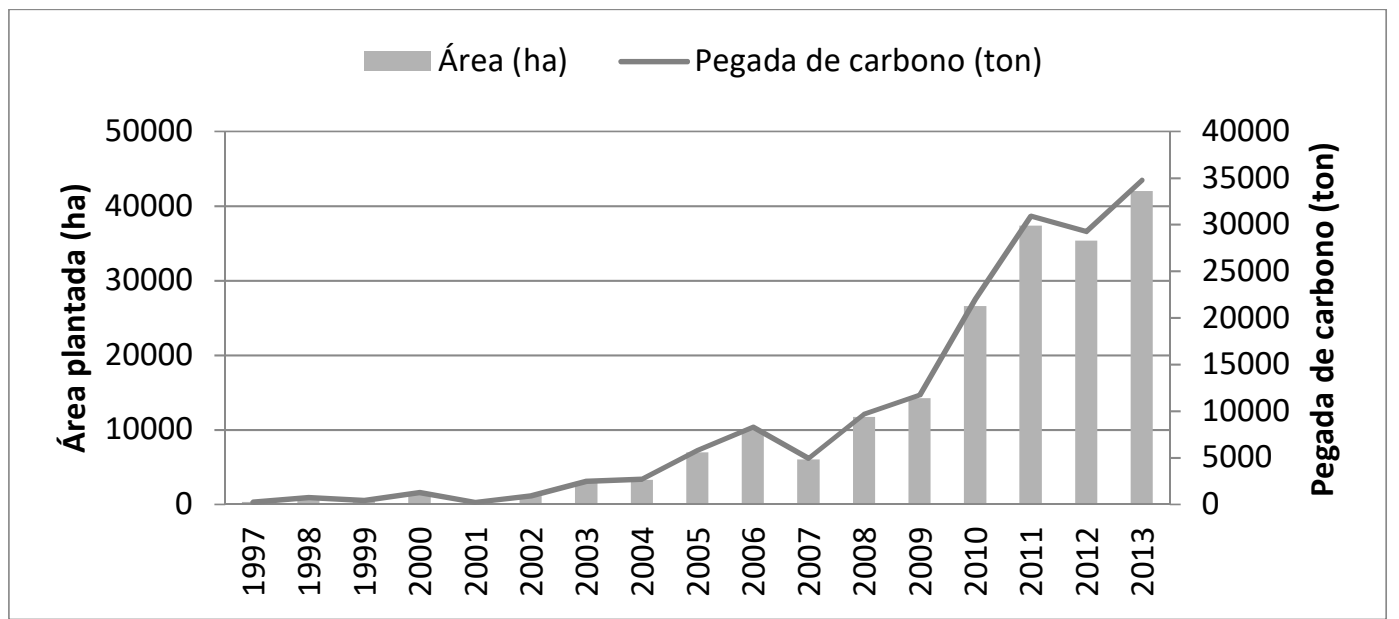

Figura 1: Pegada de carbono por total de área plantada de soja. Fonte: Autores (2015), IBGE (2013).

Comparando com os resultados obtidos por Cheng et al. (2011), a intensidade em produção média para o período de 1993 a 2007 foi de 0,110 tonC/ton enquanto que a média no município de Paragominas, no período entre 1997 a 2013, foi de 0,221 tonC/ton. Caso a produção de soja consiga ser realizada em uso algum de irrigação, o valor médio da intensidade de carbono por produção cai drasticamente, ficando em torno de 0,015 . Com isso, constata-se que o grande contribuinte do valor de PC na produção de soja em Paragominas seria a irrigação, representando $95 \%$ do valor anual.

Se detendo somente na análise da intensidade de carbono na produção no município de Paragominas, em conjunto com a área plantada e produção de soja, temos a Figura 2. A partir de sua análise, 
percebe-se a constância da intensidade de produção de carbono em valores entre 0,2 e 0,4 quando a produção de soja possui valores brutos superiores ao valor de área plantada. Os dados de intensidade que destoam dos restantes e podem ser considerados outlier são visualizados nos anos de 1998 e 2000, anos em que, conforme Tabela 2, apresentaram valores brutos de produção menores do que sua respectiva área plantada.

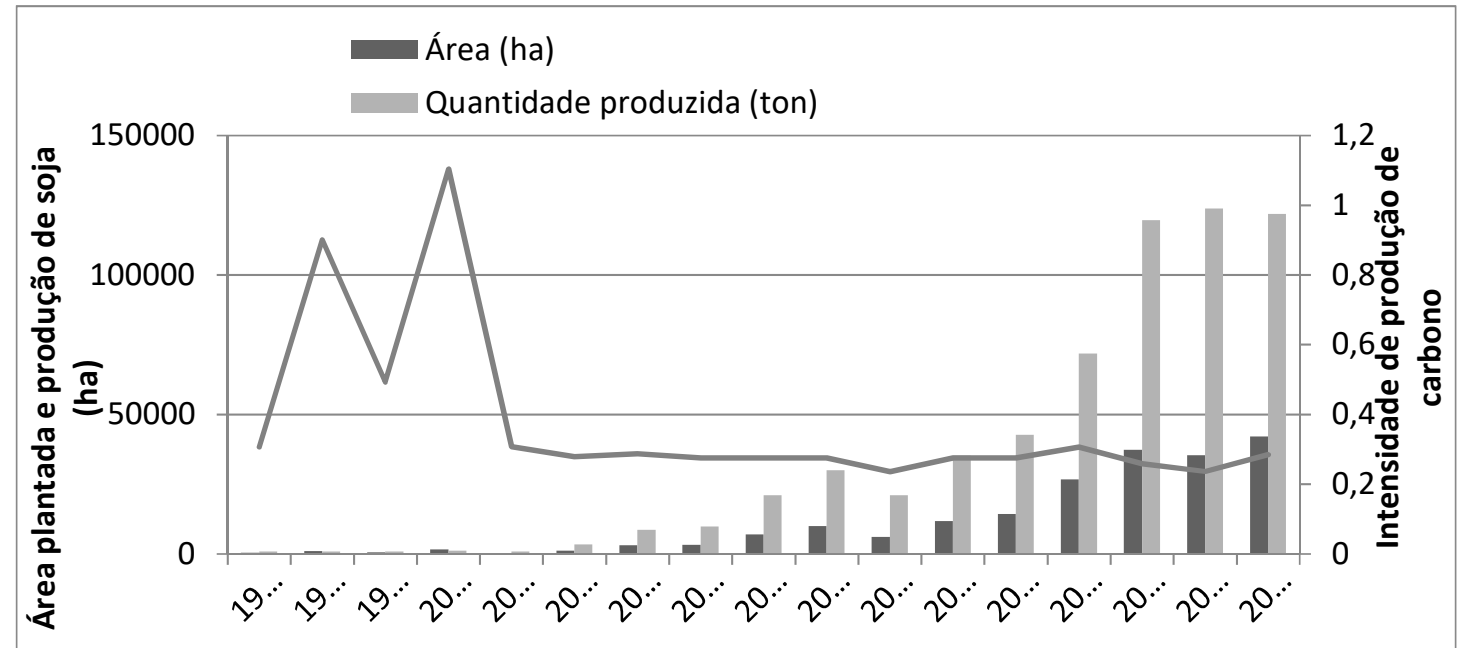

Figura 2: Comportamento da intensidade produção de carbono. Fonte: Autores (2015), IBGE (2013).

Uma contribuição importante a ser frisada é evidenciada na Figura 3. Nela percebe-se, a princípio, a óbvia correlação entre a área plantada e produtividade de soja. Contudo, ao analisar-se ano a ano, vê-se que em alguns destes houve uma produtividade menor do que a área plantada.

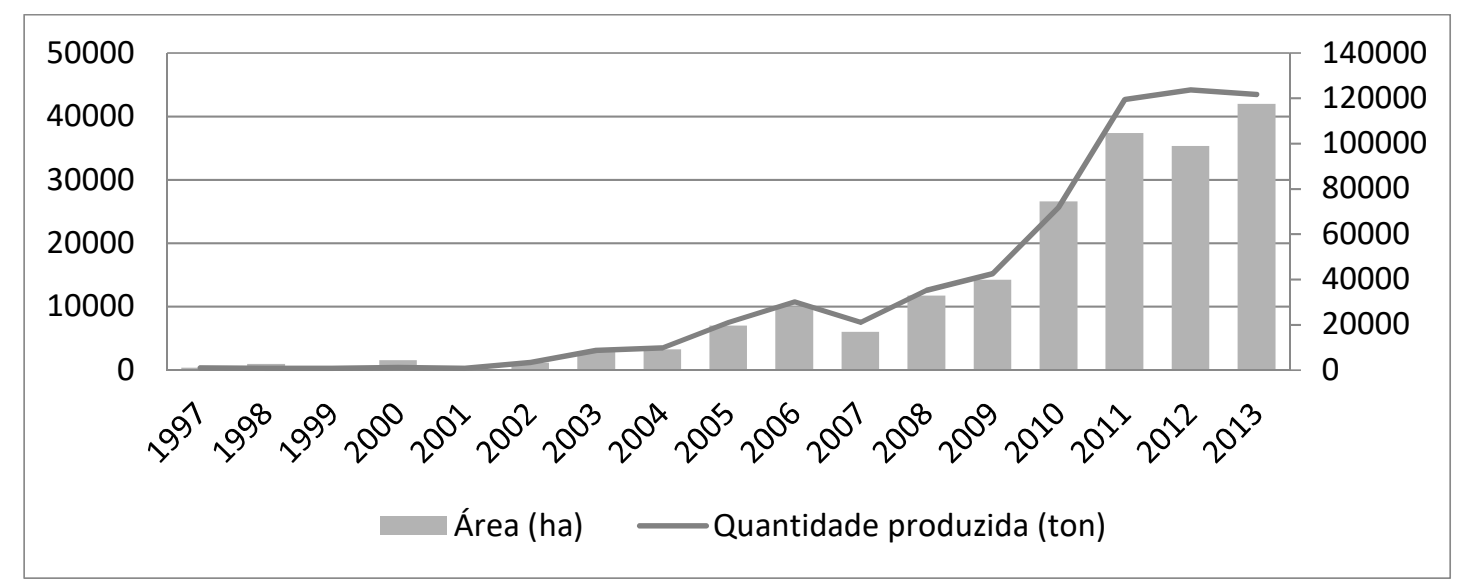

Figura 3: Produção de soja por área plantada. Fonte: IBGE, 2013.

Contudo, o ponto a ser destacado é que o inverso também é possível de acontecer, tendo-se uma linha de produção de soja consideravelmente maior do que a área plantada no ano de 2012. Este fato é de suma importância para a diminuição da pegada de carbono, haja vista que está intimamente relacionada à área plantada (Tabela 1). Deve-se, portanto, procurar se repetir esta taxa de produtividade de soja graficamente maior do que sua área plantada para que a pegada de carbono seja considerada eficiente. 


\section{CONCLUSÕES}

O uso da metodologia proposta se mostra interessante de ser utilizada por governantes pelo principal motivo de apresentar custo zero e sem necessidade de fazer análises in loco, análise de carbono no solo e de carbono emitido pela conversão de uso do solo para a atividade de soja, variáveis estas que são necessárias para o cálculo segundo outras metodologias presentes na literatura.

Ao se analisar os resultados obtidos através de tal ferramenta, foi possível investigar a realidade local quanto à emissão de GEE pela produção municipal de soja, verificando e comparando o nível de sustentabilidade da emissão de carbono ao longo de uma análise temporal. Tal análise apontou a oportunidade que o município tem de diminuir o seu valor de intensidade de carbono de 0,376 (valor médio) para 0,236 (menor valor verificado, ocorrendo no ano de 2007), bastando ocorrerem iniciativas que tentem reproduzir as mesmas condições de produção daquelas realizadas no ano em questão. Em períodos de precipitação elevada, o não uso da irrigação auxilia na redução drástica da pegada de carbono, evidenciando que as condições ambientais regionais da Amazônia acabam por favorecer a produção de soja neste sentido.

O cálculo de estimativas de pegada de carbono evidencia que as principais variáveis estão direta ou indiretamente ligadas à área plantada. Tais resultados podem servir para planejamento de ações tanto no setor da soja para os tomadores de decisão do município de Paragominas, como para discussões a respeito de mudanças climáticas e de emissões de carbono.

\section{REFERÊNCIAS}

BAYER, C.; MARTIN-NETO, L.; MIELNICZUK, J.; PAVINATO, A. Armazenamento de carbono em frações lábeis da matéria orgânica de um Latossolo Vermelho sob plantio direto. Pesquisa Agropecuária Brasileira, v. 39, n. 7, 2004.

BISPO, C. J. C.. Balanço de água em área de cultivo de soja leste da Amazônia. 2007. 148 f. Dissertação (Mestrado em Ciências Ambientais) - Universidade Federal do Pará, Belém, 2007.

CARBON TRUST. Carbon Footpirnting. 2012.

CHENG, K.; PAN, G.; SMITH, P.; LUO, T.; LI, L.; ZHENG, J.; ZHANG, X.; HAN, X.; YAN, M.. Carbon footprint of China's crop production - An estimation using agro-statistics data over 1993-2007. Agriculture, Ecosystems and Environment, Vol. 142, pp. 231-237, 2011.

CONCEIÇÃO, P.C.; DIECKOW, J.; BAYER, C.. Combined role of no-tillage and cropping systems in soil carbon stocks and stabilization. Soil \& Tillage Research, v. 129, p. 40-47, 2013.

COSTA, R. R. S.. Gestão para resultados: como gerar indicadores de gestão de pessoas, utilizando ferramentas básicas de TI. In: CONGRESSO CONSAD DE GESTÃO PÚBLICA, 6, 2013, Brasília. Anais..

CUCEK, L; KLEMES, J. J.; KRAVANJA, Z.. A Review of Footprint analysis tools for monitoring impacts on sustainability. Journal of Cleaner Production, vol. 34, p. 9-20, 2012.
DON, A.; SCHUMACHER, J.; FREIBAUER, A.. Impact of tropical land-use change on soil organic carbon stocks - a metaanalysis. Global Change Biology, v.17, n.4, 2011.

FAGUNDES, M. B. B.; ARAÚJO, C. G.; MORTARI, V. S.; REBELO, F. S.; RODRIGUES, P.. Análise dos efeitos da intervenção governamental na competitividade da produção de soja em grãos no Mato Grosso do Sul. Revista Gestão \& Regionalidade, vol. 29, n. 87, 2013.

GALLI, A.; WIEDMANN, T.; ERCIN, E.; KNOBLAUCH, D.; EWING, B.; GILJUM, S.. Integrating ecological, carbon and water footprint into a "Footprint Family" of indicators: definition and role in tracking human pressure on the planet. Revista Ecological Indicators, vol. 16, p.100-112, 2012.

GROSSI, M. C.; CHAVES, E.; JUSTINO, F. B.; SILVA, R. F. Influência das mudanças climáticas na produtividade da soja na Amazônia Legal. In: Congresso Brasileiro De Meteorologia, 26, 2010, Belém. Anais... Belém: 2010.

GUO, L. B.; GIFFORD, R. M.. Soil carbon stocks and land use change: a meta analysis. Global Change Biology, v.8, n.4, 2002.

HERTWICH, E.; PETERS, G. P.. Carbon Footprint of Nations: A Global, Trade-Linked Analysis. Environmental Science \& Technology, Vol. 43, no. 16, 2009.

HOUGHTON, R. A. ; HOUSE, J. I. ; PONGRATZ, J. ; VAN DER WERF, G. R. ; DEFRIES, R. S. ; HANSEN, M. C. ; 
LE QUÉRÉ C. ; RAMANKUTTY, N.. Carbon emissions from land use and land-cover change. Biogeosciences, v.9, n.12, 2012.

IBGE. Instituto Brasileiro de Geografia e Estatística. Indicadores de Desenvolvimento Sustentável - Brasil 2012. 2012.

IBGE. Instituto Brasileiro de Geografia e Estatística. Produção Agrícola Municipal 2013. 2013.

KAHRL, F.; LI, Y.J.; SU, Y.F.; TENNIGKEIT, T.; WILKES, A.; XU, J. C.. Greenhouse gas emissions from nitrogen fertilizer use in China. Environ. Sci. Policy, n. 13, p. 688-694, 2010.

LEÃO, R. N. R.; SOUZA, P. J. O. P.. Impactos do aquecimento global na produtividade da soja para a região de Paragominas-PA, utilizando modelagem agrometeorológica. In: Seminário Anual de Iniciação Científica da Universidade Federal Rural da Amazônia, 10., 2012, Belém. Anais... Belém: 2012.

MONTIBELLER, G.; SOUZA, G. C.; BÔLLA, K. D. S.. Economia Ecológica e Sustentabilidade Socioambiental. Revista Brasileira de Ciências Ambientais, v.23, 2012.

PACHECO, N. A.; BASTOS, T. X.; HUSNY, J. C. E.. Identificação de período de risco climático para a semeadura da soja no município de Paragominas, PA. In: Congresso Brasileiro de Meteorologia, 2004, Fortaleza. Anais do XIII Congresso Brasileiro de Meteorologia. Fortaleza: SBMET.
PANDEY, D.; AGRAWAL, M.; PANDEY, J. S.. Carbon Footprint: current methods of estimation. Environmental Monitoring and Assessment, Vol. 178, no. 1, pp. 135-160, 2011.

POST, W. M.; KWON, K. C.. Soil carbon sequestration and land-use change: processes and potential. Global Change Biology, v.6, n.3, 2000.

SANTOS, A. P. R. A.. Características agrometeorológicas da cultura de soja (Glycine max (L.) Merrill) variedade Candeias em Paragominas - PA. 2008. 124 f. Dissertação (Mestrado em Ciências Ambientais) - Universidade Federal do Pará, Belém, 2008.

SOUSA, L. L.. A logística da soja na fronteira agrícola norte e nordeste. Piracicaba, 2012. Disponível em:

<http://esalqlog.esalq.usp.br/files/biblioteca/arquivo3939.P DF>. Acesso em: 2 mai. 2015.

VEZZANI, F. M.; MIELNICZUK, J.. Agregação e estoque de carbono em argissolo submetido a diferentes práticas de manejo agrícola. Revista Brasileira de Ciência do Solo, v. 35, 2011.

WEST, T. W.; MARLAND, G.. A synthesis of carbon sequestration, carbon emissions, and net carbon flux in agriculture: comparing tillage practices in the United States. Agriculture, Ecosystems \& Environment, v. 91, n 1-3, 2002.

WEST, T. W.; MARLAND, G.. Net carbon flux from agriculture: Carbon emissions, carbon sequestration, crop yield, and land-use change. Biogeochemistry, v. 63, n. 1, p. 73-83, 2003. 